\title{
Geometrization of Classical Wave Fields
}

\author{
Oleg A.Olkhov \\ N.N.Semenov Institute of Chemical Physics, Kosygin str.,4, 119991 Moscow, Russia; olega@gagarinclub.ru
}

AIP Conf.Proc.V.962. p.316, QUANTUM THEORY: Reconsideration of Foundations-4, Vaxjo, Sweden, 11-16 June 2007 (http://proceedings.aip.org/proceedings)

\begin{abstract}
New geometrical interpretation of quantum physics is suggested. It is shown that the Dirac equation for free quantum particles can be considered as a relation describing propagation of the space topological defect with wave and corpuscular properties. Such interpretation explains all properties of quantum formalism that seems "strange"within classical representations: appearance of probabilities, absence of the particles trajectories, unremovable influence of the measurement procedure, instantaneous nonlocal correlation in EPR-experiments. The same interpretation is suggested for the Maxwell equations for free electromagnetic waves. This interpretation explains independence of light velocity on the source movement.
\end{abstract}

Keywords: quantum mechanics, geometrization, topological defects, EPR-paradox, many-body problem PACS: 03.65.Ca, 03.65.Ta

\section{INTRODUCTION}

We will show within unified approach, that the free matter field (quantum particle with spin $1 / 2$ ) and free electromagnetic waves can both be considered not as something existing into the space that plays the role of scene but as a propagating topological defects of the space itself. These defects appear to be embedded into five-dimensional space, and observable quantum object and electromagnetic wave are intersections of the defects with the three-dimensional physical space. Their wave properties are a consequence of the periodical movement of the defect in the "outer"space-just this movement attributes phase to the particle and so defines its wave properties. The classical notions of mass, energy and momentum are expressed through the parameters with dimensionality of length, and the Planck constant and the light velocity play the role of coefficients of transfer from one system of units to the other. It is shown that light velocity can be considered as a topological invariant, and this is the reason for its independence of the source motion. The uncertainty of shapes of topological manifolds is the reason of the appearance of probabilistic description.

We start with the geometrization of matter field and, doing so, we have to keep in mind the exceptional accuracy of the modern quantum formalism. Therefore, we suppose that attempts to find out new geometrical description of quantum objects have to begin not with the creation of a new formalism but with finding out a geometrical interpretation of the well-known quantum equations whose validity is beyond question. This is the reason why we start with an attempt to find out geometrical interpretation of the Dirac equation for free particle. Preliminary results were published earlier [1].

\section{TOPOLOGICAL INTERPRETATION OF THE DIRAC EQUATION}

This equation has the following symbolic form (see, e.g.,[2])

$$
i \gamma^{\mu} \partial_{\mu} \psi=m \psi
$$


where $\partial_{\mu}=\partial / \partial x_{\mu}, \quad \mu=1,2,3,4, \quad \psi(x)$ is the four-component Dirac bispinor, $x_{1}=t, x_{2}=x, x_{3}=y$, $x_{4}=z$, and $\gamma^{\mu}$ are four-row Dirac matrices. The summation in Eq.(1) goes over the repeating indices with a signature $(1,-1,-1,-1)$. Here, $\hbar=c=1$. For definite values of 4 -momentum $p_{\mu}$, the solution to Eq.(1) has the form of the plane wave

$$
\psi=u\left(p_{\mu}\right) \exp \left(-i p_{\mu} x^{\mu}\right)
$$

where $u\left(p_{\mu}\right)$ is a normalized bispinor. Substitution of (2) in Eq.(1) gives:

$$
p_{1}^{2}-p_{2}^{2}-p_{3}^{2}-p_{4}^{2}=m^{2}
$$

Different wave fields are described by different tensors: they realize different representations of the Lorentz group (group of 4-rotation). Dirac bispinor consists of two-component spinors that realizes two-dimensional representation of the Lorentz group, and this is the formal reason to classify the Dirac wave field of Eq.(1) as the object with "internal"angular momentum whose Z-projection equals to $\pm 1 / 2$ [3]. Let us, firstly, consider transformation properties of the Dirac bispinor from the other point of view. It is known that there may be established correspondence between every kind of tensors and some class of geometrical objects in the sense that these tensors define invariant properties of above objects. For example, usual vectors correspond to simplest geometrical objects-to points [4], and this is the reason why Newtonian mechanics uses vectors within its formalism. From this point of view spinors correspond to nonorientable geometrical object (see, e.g., [5]). Therefore, we suppose that spinors are used in Eg.(1), because this equation describes some nonorientable geometrical object and "spin $=1 / 2$ "is a formal expression of the nonorientable property of the object.

The above assumption have to be considered as the starting hypothesis only. To define properties of the proposed geometrical object more exactly we consider more precisely the symmetry properties of the solution of Eq.(1). We rewrite function (2) and relation (3) in the form

$$
\begin{gathered}
\psi=u\left(p_{\mu}\right) \exp \left(-2 \pi i x^{\mu} \lambda_{\mu}^{-1}\right) . \\
\lambda_{1}^{-2}-\lambda_{2}^{-2}-\lambda_{3}^{-2}-\lambda_{4}^{-2}=\lambda_{m}^{-2}, \quad \lambda_{\mu}=2 \pi p_{\mu}^{-1}, \quad \lambda_{m}=2 \pi m^{-1} .
\end{gathered}
$$

Let us now consider $\lambda_{\mu}$ as some parameters with a dimensionality of length that have nothing to do with any wave process into the space. Function (4) is an invariant with respect to coordinates transformations

$$
x_{\mu}^{\prime}=x_{\mu}+n_{\mu} \lambda_{\mu}, \quad n_{\mu}=0, \pm 1, \pm 2, \ldots .
$$

Transformations (6) can be considered as elements of the discrete group of translations operating in the 4 -space where wave function (4) is defined. Then function (4) can be considered as a vector realizing this group representation.

As a bispinor, function (4) realizes representation of one more group of the symmetry transformation of 4 -space. Being a four-component spinor, $\psi(x)$ is related to the matrices $\gamma^{\mu}$ by the equations (see, e.g. [3])

$$
\psi^{\prime}\left(x^{\prime}\right)=\gamma^{\mu} \psi(x)
$$

where $x \equiv\left(x_{1}, x_{2}, x_{3}, x_{4}\right)$, and $x^{\prime} \equiv\left(x_{1},-x_{2},-x_{3},-x_{4}\right)$ for $\mu=1, x^{\prime} \equiv\left(-x_{1}, x_{2},-x_{3},-x_{4}\right)$ for $\mu=2$, and so on. This means that the matrices $\gamma^{\mu}$ are the matrix representation of the group of reflections along three axes perpendicular to the $x_{\mu}$ axis, and the Dirac bispinors realize this representation [6].

Taken together, above two groups form a group of four sliding symmetries with perpendicular axes (sliding symmetry means translations plus corresponding reflections [7]). The physical space-time does not have such symmetry.So, this group may operate only in some auxiliary space. From the other hand, it is known that discrete groups operating in some space can reflect a symmetry of geometrical objects that have nothing in common with this space. It will be the case when such space is a universal covering space of some closed topological manifold. Universal covering spaces are auxiliary spaces that are used in topology for the description of closed manifolds, because discrete groups operating in these spaces are isomorphic to fundamental groups of manifolds-groups whose elements are different classes of closed pathes on manifolds (so called $\pi_{1}$ group [8,9]). And we assume now, that function (4) realizes a representation of the 
fundamental group of some closed nonorientable topological 4-manifold-a specific curved part of the spacetime. Eq.(1) describes this manifold and imposes limitations (5) on the possible values of the fundamental group parameters $\lambda_{\mu}$. Space-time plays also the role of an universal covering space for above manifold.

How above manifold can describe something moving in the space ?. At the present time, only twodimensional euclidean closed manifolds are classified in details, and their fundamental groups and universal covering planes are identified [8]. Therefore, we have no opportunity for rigorous consideration of specific properties of suggested psudoeuclidean 4-manifold. But qualitative properties, explaining main ideas of new interpretation, can be investigated using one of the advantages of geometrical approach-possibility of employment of low-dimensional analogies. Using these analogies we will show within elementary topology that above mentioned 4-manifold represents propagation of the space topological defect that can demonstrate specific properties of quantum particle represented by solution (2): stochastic behavior and wave-corpuscular dualism.

\section{STOCHASTIC BEHAVIOR}

Consider the simplest example of closed topological manifolds-one-dimensional manifold homeomorphic to a circle with given perimeter length $\lambda$. The closed topological manifold is representable by any of its possible deformations (without pasting) that conserve manifold's continuity, and we will see that just this property explains appearance of probabilities in quantum formalism. For simplicity we consider plane deformations of the circle(some of the possible deformations are shown at Fig.1).

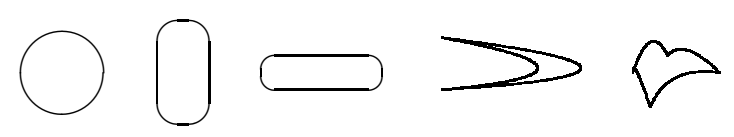

FIGURE 1. Stochastic behavior.

To use concrete simple calculations, we consider only all possible manifold's deformations that have a shape of ellipse with perimeter length $\lambda$. The equation for the ellipse on an euclidean plane has the form

$$
x^{2} / a^{2}+y^{2} / b^{2}=1,
$$

where all possible values of the semiaxes $a$ and $b$ are connected with the perimeter length $\lambda$ by the known approximate relation

$$
\lambda \simeq \pi\left[1,5(a+b)-(a b)^{1 / 2}\right] .
$$

This means that the range of all possible values of $a$ is defined by the inequality $a_{\min } \leq a \leq a_{\max } \simeq$ $\lambda / 1,5 \pi, \quad a_{\min } \ll a_{\max }$.

In the pseudoeuclidean two-dimensional "space-time,"the equation for our ellipses has the form (after substitution $y=i t)$

$$
x^{2} / a^{2}-t^{2} / b^{2}=1,
$$

and this equation defines the dependence on time $t$ for a position of the point $x$ of the manifold corresponding to definite $a$. At $t=0, x= \pm a$; that is, our manifold is represented by the two point sets in one-dimensional euclidean space, and the dimensions of these point sets are defined by all possible values of $a$. So, at $t=0$, the manifold is represented by two regions of the one-dimensional euclidean space $a_{\min } \leq|x|=a \leq a_{\max }$. It can easily be shown that at $t \neq 0$ these regions increase and move along the $\mathrm{x}$-axis in opposite directions 
(at Fig.2 this movement is shown only for positive direction).

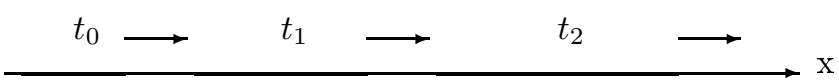

FIGURE 2. Propagation of the spreading region of the space.

All another possible deformations of our circle will be obviously represented by points of the same region, and every such point can be considered as a possible position of the "quantum object"described by our manifold. All manifold's deformations are realized with equal probabilities (there are no reasons for another suggestion). Therefore, all possible positions of the point-like object into the region are realized with equal probabilities. So, this example shows the possibility of the consideration of above object as a point with probability description of its positions as it suggested within standard representation of quantum particles. In fact, this point is yet a geometrical point only. Below we will show, how this point becomes the material point. Note also that parameter $\lambda$ defines the minimal size of a region $\Delta x \sim \lambda$, where the object can be localized (region at $t=0$ ).

\section{TOPOLOGICAL DEFECT. WAVE CORPUSCULAR PROPERTIES}

Above example does not explain what geometrical properties allow to differ points of the moving region from neighbour points of the euclidean space making them observable. To answer at this question we consider more complex analogy of the closed 4-manifold-two-dimensional torus. In euclidean 3 -space such torus is denoted as topological production of two circles $-S^{1} \times S^{1}$. The role of different manifold's deformations as a reason for stochastic behaviour was considered in Section 2. Therefore, now we restrict our consideration to one simplest configuration when one of $S^{1}$ is a circle in the plane $X Y$ and another is a circle in the plane $Z X$ (we denote it as $S_{1}^{\prime}$ ).

In pseudueuclidean space this torus looks like a hyperboloid. The hyperboloid appears if we replace the circle $S_{1}^{\prime}$ by a hyperbola (as it was done in Section 2). Positions of the geometrical object described by our pseudoeucliden torus are defined by time cross-sections of the hyperboloid. These positions form an expanding circles in the two-dimensional euclidean plane (Fig.3).

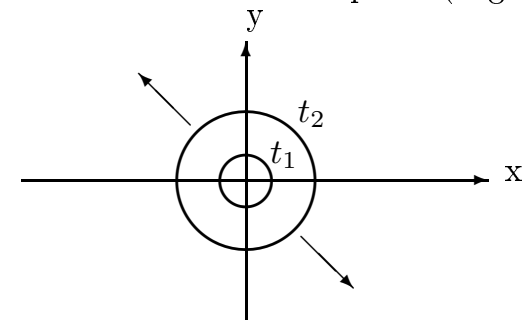

FIGURE 3. Topological defect.

But we need to have in mind that two-dimensional pseudoeuclidean torus describes the object existing into two-dimensional space-time with one-dimensional euclidean "physical"space. This means that an observable part of the object is represented in our example by the points of intersections of above circle with $0 X$ axis though, as a whole, the circle is "embedded"into two-dimensional, "external"space. This circle can be considered as a topological defect of the physical one-dimensional euclidean space. Just an affiliation of the intersection points to the topological object differs these points geometrically from neighboring points of the one-dimensional euclidean space. So, in pseudoeuclidean four-dimensional physical space-time the suggested object described by the Dirac equation looks like a topological defect of physical euclidean 3-space that is embedded into 5-dimensional euclidean space, and its intersection with physical space represents an observable quantum object.

Above analogy with torus does not yet demonstrate appearance of any wave-corpuscular properties of the object, represented in "physical"one-dimensional space by the moving intersection point- properties 
that could be expressed by wave function (5) and relation (4). In the case of considered two-dimensional "space-time"this solution has the form

$$
\psi=u(p) \exp \left(-2 \pi i x^{1} \lambda_{1}^{-1}+2 \pi i x^{2} \lambda_{2}\right) .
$$

Topological defect represented by the expanding circle does not demonstrate any periodical movements when the intersection point (physical object) propagates along one-dimensional euclidean $0 X$-space.

We will see below that appearance of observable wave-corpuscular properties is a consequence of nonorientable character of the topological defect. Torus is a orientable two-dimensional closed manifold and, therefore, we need to use some nonorientable low-dimensional analogy. The nonorientable Klein bottle could be such two-dimensional analogy [6,7]. In the case with torus topological defect was represented by cross-sections of pseudoeuclidean torus-plane circles. The Klein bootle is a manifold that is obtained by gluing of two Mobius strips [10]. Therefore, the Klein bootle cross-section is an edge of the Mobius strip. This edge can not be placed in the two-dimensional $X Y$-plane without intersections, and it means that corresponding topological defect is now a closed curve embedded into three-dimensional $X Y Z$-space.

In this case the position of the topological defect relative to its intersection with $0 X$ axis (physical object) can change periodically. Parameters of this periodical movement depend on geometrical parameters $\lambda_{1}, \lambda_{2}$ (there are no other parameters with corresponding dimensionality). Such periodical process can be expressed by the function (10). It leads to the new interpretation of the wave function as a description of periodical movement of the topological defect relative to its projection on the physical space.

In this case corpuscular properties of the above periodical movement appear as a result of the definition for classical notion of 4-momentum trough the wave characteristic of the topological object, namely

$$
p_{\mu}=2 \pi / \lambda_{\mu} .
$$

Substitution of these relations into (18) leads to the Dirac solution (2)

$$
\psi=u(p) \exp \left(-i p_{1} x^{1}+i p_{2} x^{2}\right) .
$$

It is important to note that within suggested geometrical interpretation the notions of the less general, macroscopic theory (4-momentums) are defined by (11) trough the notions of more general microscopic theory (wave parameters of the defect periodical movement). This looks more natural than the opposite definitions (4) within traditional interpretation.

\section{GEOMETRIZATION OF ELECTROMAGNETIC WAVES}

Let us write the Maxwell equations for electromagnetic waves in vacuum in the symbolic form analogous to the Dirac equation (1). Namely, we write these equations in the Majorana form [11]

$$
\begin{gathered}
i \frac{\partial \mathbf{f}^{+}}{\partial t}=(\mathbf{S p}) \mathbf{f}^{+}, \quad \mathbf{p \mathbf { f } ^ { + }}=0, \\
i \frac{\partial \mathbf{f}^{-}}{\partial t}=-(\mathbf{S p}) \mathbf{f}^{-}, \quad \mathbf{p \mathbf { f } ^ { - }}=0,
\end{gathered}
$$

where

$$
\mathbf{f}^{+}=\mathbf{E}+i \mathbf{H}, \quad \mathbf{f}^{-}=\mathbf{E}-i \mathbf{H} .
$$

Here $\mathbf{E}$ is an electric field, $\mathbf{H}$ is a magneyic field, $\mathbf{p}=-i \nabla$ and $\mathbf{S}$ is a vector-matrix

$$
S_{x}=\left(\begin{array}{ccc}
0 & 0 & 0 \\
0 & 0 & -i \\
0 & i & 0
\end{array}\right), \quad S_{y}=\left(\begin{array}{ccc}
0 & 0 & i \\
0 & 0 & 0 \\
-i & 0 & 0
\end{array}\right), \quad S_{z}=\left(\begin{array}{ccc}
0 & -i & 0 \\
i & 0 & 0 \\
0 & 0 & 0
\end{array}\right)
$$

It can be easily seen that Egs.(19) may be rewritten in the symbolic form analogous to the symbolic form of the Dirac equation (1)

$$
i \Gamma^{\mu} \partial_{\mu} \mathbf{f}=0
$$


where bivector $\mathbf{f}$ and matrix $\Gamma^{\mu}$ have the form

$$
\Gamma^{0}=\left(\begin{array}{cc}
0 & 1 \\
1 & 0
\end{array}\right), \quad \Gamma^{1,2,3}=\left(\begin{array}{cc}
0 & -\mathbf{S} \\
\mathbf{S} & 0
\end{array}\right), \quad \mathbf{f}=\left(\begin{array}{c}
\mathbf{f}^{+} \\
\mathbf{f}^{-}
\end{array}\right) .
$$

We write here six-row matrices trough three-row ones). We see that Egs.(16) has formally the same form as the Dirac equation (1) with $m=0$. Only instead of the Dirac bisinor $\psi$ we have here bivector $\mathbf{f}$, and instead Dirac matrices $\gamma^{\mu}$ we have matrices $\Gamma^{\mu}$. As Maxwell's Egs.(16) looks formally like Dirac's Egs.(1) it seems reasonable to use for their geometrization the same arguments as we used for the Dirac equation geometrization.

For plane waves the solution of Eq.(16) has the form

$$
\mathbf{f}^{+}=\mathbf{f}_{k}^{+} \exp i(\mathbf{k r}-\omega t), \quad \mathbf{f}^{-}=\mathbf{f}_{k}^{-} \exp i(\mathbf{k r}-\omega t), \quad \omega=|\mathbf{k}| .
$$

As for topological interpretation of solution (2) of the Dirac equation we suggest that function (17) does not define any wave process into the space but describes periodical movement of the space topological defect. As function (2) solution (17) can be also considered as the realization of fundamental group of some closed topological manifold. Due to exponential factor in (17) this group contains the translation group (as for solution (2)), but now our solution are complex bivectors-not a bispinors. These vectors does not realize the representation of reflections along three different axes as it was for bispinors. These vectors $\mathbf{f}^{+}$and $\mathbf{f}^{-}$ consist of axial and polar vectors (see (14)) and thus these vectors are transformed one into another only in result of the reflection of space axes. Therefore, solution (17) realizes representation of a sliding symmetry in 4-space only along time-axis. This distinguishes the supposed fundamental group from the fundamental group considered in previous Sections (four sliding symmetries along four perpendicular axes).

There is no investigation in topology, where 4-manifolds with above fundamental group were considered. So, we again can establish connections between geometrical properties of the manifold and observable physical properties of electromagnetic waves only using low-dimensional analogies. Wave-corpuscular dualism of electromagnetic waves and possibility of stochastic behavior can be demonstrated in the same manner as in Sections 2,3. But electromagnetic waves have some additional important property-their velocity does not depend on the source motion. We will show below how geometrical properties of the closed topological 4-manifold can explain this fact.

Suppose that 4-manifold corresponding to electromagnetic wave has the form $M^{3}(\mathbf{r}) \times M^{1}(\mathbf{r}, t)$, that is it can be represented as a product of nonorientable three-dimensional euclidean closed manifold $M^{3}(\mathbf{r})$ and one-dimensional manifold $M^{1}(\mathbf{r}, t)$ homeomorphic to a pseudoeuclidean circle. The formal reason for such representation is the distinguished role of the euclidean space within fundamental group: only into euclidean subspace translation group is combined with reflections. Consider now a low-dimensional analogy that explains an independence of light velocity on the source motion.

Instead of four-dimensional manifold $M^{3}(\mathbf{r}) \times M^{1}(\mathbf{r}, t)$ we consider, as in previous Section, two-dimensional analogy-manifold $S^{1} \times S_{1}^{\prime}$, where $S^{1}$ is a one-dimensional euclidean circle and $S_{1}^{\prime}$ is a pseudoeuclidean circle. This manifold was considered in Section 3, and it looks like a hyperboloid. For electromagnetic waves $m=0, E=c p$. Within our notation it leads to relation $p_{1}=p_{2}=p$ or $\lambda_{1}=\lambda_{2}=\lambda$. Therefore, there have to be only one parameter with dimensionality of length, and this will be the case if $S_{t}^{1}$ is a pseudoeuclidean circle of zeroth radius. Equation for such circle has the form $x^{2}-t^{2}=0$, and hyperboloid is transformed into a cone (Fig.3). This means that the points representing in this example electromagnetic wave move with velocity equals \pm 1 ( $\pm c$-in chosen units system), and this velocity does not depend on coordinate frame rotations (does not depend on transfer from one moving inertial frame to another). From topological point of view this result is a consequence of the fact that the zeroth radius can be considered as topological invariant. Therefore, within geometrical approach light velocity appears to be topological invariant of the manifold representing electromagnetic wave, and this is the reason of its independence of the source motion.

\section{NEW OPPORTUNITIES}

Let us notice shortly some new opportunities that arise within suggested topological concept. For example, including into physical world the objects from outer five-dimensional space can explain rather simply the 
origin of nonlocal correlation in EPR-experiments. We see at Fig.3 that two noninteracting identical particles (intersection points) can move in opposite directions being parts of the same topological defect (the circle). In the act of measurement of one of the particles the symmetry of the whole topological defect can be changed (for example, it can become nonorientable manifold being at first orientable one). And another particle, being the part of the same defect, would "feel"at once this change without any interaction (duration of above symmetry change is defined by measurement procedure, and it does not depend on the distance between particles).

The more important consequence of new approach is the possibility to overcome the difficulties of physics of many-electron atoms connected with the "many-body"problem. Earlier we suggested geometrical interpretation of the Dirac equation for hydrogen atom [1]. The electron Coulomb potential appears to be within this interpretation the connectivity of the universal covering space of corresponding topological defect and has nothing to do with any negative particle inside the atom. It means, may be, that there is no necessity to consider atoms as many-particle systems because many-particles potentials have to be changed within geometrical approach by corresponding connectivities, and these coonectivities are functions of only one space-time coordinate. Then new possible relativistic equations (instead of Schrodinger ones) have to be "one-particle"equations for functions of one space-time coordinate. We are now looking for such new possible potentials and equations.

\section{REFERENCES}

1. O.A. Olkhov, Journ.of Phys.:Conf.Ser.,67,012037(2007); arXiv: 0706.3461.

2. J.D. Bjorken, S.D. Drell, Relativistic Quantum Mechanics and Relativistic Quantum Fields, New York McGraw-Hill, 1964.

3. A.I. Achiezer, S.V. Peletminski, Fields and Fundamental Ineractions, Kiev Naukova Dumka, 1986, Ch.1. 4. P. L. Rachevski, Riemannian geometry and tensor analysis, Moscow, Nauka, 1966, §55.

5. V.A. Jelnorovitch, Theory of spinors and its applications, Moscow, August-Print, 2001, §1.3.

6. M. Hamermesh, Group Theory and Its Application to Physical Problems, Argonne National Laboratory, 1964.

7. H.S.M. Coxeter, Introduction to Geometry, N.Y.-London John Wiley and Sons, 1961.

8. B.A. Dubrovin, S.P. Novikov, A.T. Fomenko, Modern geometry, Moscow, Nauka, 1986.

9. A.S. Schvartz Quantum Field Theory and Topology, Moscow, Nauka, 1989.

10. D. Gilbert, S. Kon-Fossen, Nagladnaya geometriya (rus), Moscow, Nauka, 1981.

11. A.I. Achiezer, V.B. Berestetski, Quantum Electrodynamics, Moscow, Nauka, 1981. 